\title{
Existence of Pseudo-Superinvolutions of the First Kind
}

\author{
Ameer Jaber \\ Department of Mathematics, The Hashemite University, Zarqa 13115, Jordan
}

Correspondence should be addressed to Ameer Jaber, ameerj@hu.edu.jo

Received 6 March 2007; Revised 8 July 2007; Accepted 2 November 2007

Recommended by Alexander Rosa

Our main purpose is to develop the theory of existence of pseudo-superinvolutions of the first kind on finite dimensional central simple associative superalgebras over $K$, where $K$ is a field of characteristic not 2 . We try to show which kind of finite dimensional central simple associative superalgebras have a pseudo-superinvolution of the first kind. We will show that a division superalgebra $\Phi$ over a field $K$ of characteristic not 2 of even type has pseudo-superinvolution (i.e., $K$-antiautomorphism $J$ such that $\left(d_{\delta}\right)^{J^{2}}=(-1)^{\delta} d_{\delta}$ ) of the first kind if and only if $\Phi$ is of order 2 in the Brauer-Wall group $\mathrm{BW}(K)$. We will also show that a division superalgebra $\Phi$ of odd type over a field $K$ of characteristic not 2 has a pseudo-superinvolution of the first kind if and only if $\sqrt{-1} \in K$, and $\Phi$ is of order 2 in the Brauer-Wall group $B W(K)$. Finally, we study the existence of pseudo-superinvolutions on central simple superalgebras $\mathcal{A}=$ $M_{p+q}\left(\Phi_{0}\right)$.

Copyright ( 2008 Ameer Jaber. This is an open access article distributed under the Creative Commons Attribution License, which permits unrestricted use, distribution, and reproduction in any medium, provided the original work is properly cited.

\section{Introduction}

Let $K$ be a field of characteristic not 2. An associative superalgebra is a $\mathbb{Z}_{2}$-graded associative $K$ algebra $\mathcal{A}=\mathcal{A}_{0}+\mathcal{A}_{1}$. A superalgebra $\mathcal{A}$ is central simple over $K$, if $\widehat{Z}(\mathcal{A})=K$, where $(\widehat{Z}(\mathcal{A}))_{\alpha}=$ $\left\{a_{\alpha} \in \mathcal{A}_{\alpha} \mid a_{\alpha} b_{\beta}=(-1)^{\alpha \beta} b_{\beta} a_{\alpha}\right.$ for all $\left.b_{\beta} \in \mathcal{A}_{\beta}\right\}$, and the only superideals of $\mathcal{A}$ are $(0)$ and $\mathcal{A}$.

Finite dimensional central simple associative superalgebras over a field $K$ are isomorphic to End $V \cong M_{n}(\boldsymbol{\Phi})$, where $\boldsymbol{\Phi}=\Phi_{0}+\Phi_{1}$ is a finite dimensional associative division superalgebra over $K$, that is, all nonzero elements of $\Phi_{\alpha}, \alpha=0,1$, are invertible, and $V=V_{0}+V_{1}$ is an $n$-dimensional $\Phi$ superspace.

If $\boldsymbol{\Phi}_{1}=\{0\}$, the grading of $M_{n}(\boldsymbol{\Phi})$ is induced by that of $V=V_{0}+V_{1}, \mathcal{A}=M_{p+q}(\boldsymbol{\Phi})$, $p=\operatorname{dim}_{\circledast} V_{0}, q=\operatorname{dim}_{\circledast} V_{1}$, so $p+q$ is a nontrivial decomposition of $n$. While if $\Phi_{1} \neq\{0\}$, then the grading of $M_{n}(\Phi)$ is given by $\left(M_{n}(\Phi)\right)_{\alpha}=M_{n}\left(\Phi_{\alpha}\right), \alpha=0,1$, as we recall. 
Let $\mathcal{A}=\mathcal{A}_{0}+\mathcal{A}_{1}$ be any associative superalgebra over a field $K$ of characteristic not 2 , and let $*: \mathcal{A} \rightarrow \mathcal{A}$ be an antiautomorphism on $\mathcal{A}$, then $*$ is called a pseudo-superinvolution on $\mathcal{A}$ if $\left(a_{0}+b_{1}\right)^{* *}=a_{0}-b_{1}$.

In recent work on the representations of Jordan superalgebras which has yet to appear, Martinez and Zelmanov make use of pseudo-superinvolutions.

We recall a theorem of Albert which shows that a finite dimensional central simple algebra over a field $k$ has an involution of the first kind if and only if it is of order 2 in the Brauer group $\mathrm{Br}(k)$. The proof of this classical theorem is in many books of algebra, for example, see [1, Chapter 8, Section 8].

Throughout my work on the existence of superinvolutions of the first kind which has yet to appear, we prove that finite dimensional central simple division superalgebras of odd or even type with nontrivial grading over a field $K$ of characteristic not 2 have no superinvolutions of the first kind, also these results were introduced in [2, Proposition 9], [3]. Moreover, we introduce an example of a central simple superalgebra $\mathcal{A}=M_{n}(\boldsymbol{\Phi})$ over a field $K$ of characteristic not 2, where $\Phi_{1} \neq\{0\}$, such that $\mathcal{A}$ has no superinvolution of the first kind, but it is of order 2 in the Brauer-Wall group BW $(K)$, which means that Albert's theorem does not hold for superinvolutions and this is one of the reasons why one introduces a generalization for which it does.

In [2, Theorem 7], Racine proved that $\mathcal{A}=M_{n}(\boldsymbol{\oplus})$ has a superinvolution if and only if $\Phi$ has. Therefore, if $\mathcal{A}$ is a finite dimensional central simple associative superalgebra over a field $K$ of characteristic not 2 such that $\mathcal{A}$ has a superinvolution of the first kind, then $\mathcal{A}=M_{p+q}(\mathscr{\Phi})$, where $\Phi$ is a division algebra over $K$.

Let $\theta$ be a division superalgebra with nontrivial grading over a field $K$ of characteristic not 2. Since if $\mathbb{A}$ is a central simple associative superalgebra over $K$, then by [2, Theorem 3] $\mathscr{A}=M_{n}(\boldsymbol{\Phi})$, where $\Phi_{1} \neq\{0\}$ or $\mathscr{A}=M_{p+q}(\boldsymbol{D})$, where $\boldsymbol{\Phi}_{1}=\{0\}$. In Section 2 , we give some basic definitions for the supercase.

In Section 3, we classify the existence of pseudo-superinvolution of the first kind on $\Phi$ and we prove the following results.

(1) If $\mathscr{A}=M_{n}(\Phi)$, where $\Phi_{1} \neq\{0\}$, then $\mathcal{A}$ has a pseudo-superinvolution of the first kind if and only if $\Phi$ has. Therefore, it is enough to classify the existence of a pseudosuperinvolution of the first kind on $\Phi$.

(2) A division superalgebra $\Phi$ of even type over a field $K$ of characteristic not 2 has a pseudosuperinvolution of the first kind if and only if $\Phi$ is of order 2 in the Brauer-Wall group $\mathrm{BW}(K)$.

(3) A division superalgebra $\Phi$ of odd type over a field $K$ of characteristic not 2 has a pseudosuperinvolution of the first kind if and only if $\sqrt{-1} \in K$ and $\Phi$ is of order 2 in the BrauerWall group BW $(K)$.

In Section 4, we classify the existence of a pseudo-superinvolution of the first kind on $\mathcal{A}=$ $M_{p+q}(\Phi)$, where $\Phi$ is a division algebra over $K$.

Finally, if $K$ is a field of characteristic 2 , and $\mathcal{A}$ is a central simple associative superalgebra over $K$, then a superinvolution (which is a pseudo-superinvolution) on $\mathcal{A}$ is just an involution on $\mathcal{A}$ respecting the grading. Moreover, if $\mathcal{A}$ is of order 2 in the Brauer-Wall group BW $(K)$, then the supercenter of $\mathcal{A}$ equals the center of $\mathcal{A}$ and $\widehat{\otimes}_{K}=\otimes_{K}$, which means that $\mathcal{A}$ is of order 2 in the Brauer group $\operatorname{Br}(K)$. Thus, by theorem of Albert, $\mathcal{A}$ has an involution of the 
first kind, but since $\mathcal{A}$ is of order 2 in the Brauer-Wall group BW $(K), \mathcal{A}$ has an antiautomorphism of the first kind respecting the grading, therefore by [1, Chapter 8 , Theorem 8.2$], \mathcal{A}$ has an involution of the first kind respecting the grading, which means that $\mathbb{A}$ has a superinvolution (which is a pseudo-superinvolution) of the first kind if and only if $\mathbb{A}$ is of order 2 in the Brauer-Wall group BW $(K)$.

\section{Basic definitions}

Definition 2.1. If $R=R_{0}+R_{1}$ is an associative super-ring, a (right) $R$-supermodule $M$ is a right $R$-module with a grading $M=M_{0}+M_{1}$ as $R_{0}$-modules such that $m_{\alpha} r_{\beta} \in M_{\alpha+\beta}$ for any $m_{\alpha} \in M_{\alpha}, r_{\beta} \in R_{\beta}, \alpha, \beta \in \mathbf{Z}_{2}$. An $R$-supermodule $M$ is simple if $M R \neq\{0\}$ and $M$ has no proper subsupermodule.

Following [2], we have the following definition of $R$-supermodule homomorphism.

Definition 2.2. Suppose that $M$ and $N$ are $R$-supermodules. An $R$-supermodule homomorphism from $M$ into $N$ is an $R_{0}$-module homomorphism $h_{\gamma}: M \rightarrow N, \gamma \in \mathbf{Z}_{2}$, such that $M_{\alpha} h_{\gamma} \subseteq N_{\alpha+\gamma}$ and

$$
\left(m_{\alpha} r_{\beta}\right) h_{\gamma}=\left(m_{\alpha} h_{\gamma}\right) r_{\beta}, \quad \forall m_{\alpha} \in M_{\alpha}, r_{\beta} \in R_{\beta}, \alpha, \beta \in \mathbf{Z}_{2}
$$

Definition 2.3. The opposite super-ring $R^{\circ}$ of the super-ring $R$ is defined to be $R^{\circ}=R$ as an additive group, with the multiplication given by

$$
b_{\beta} \circ c_{\gamma}:=(-1)^{\beta \gamma} c_{\gamma} b_{\beta}, \quad b_{\beta} \in R_{\beta}, c_{\gamma} \in R_{\gamma} .
$$

So if $\mathcal{A}$ is a superalgebra, then $\mathcal{A}^{\circ}$ is just the opposite super-ring of $\mathcal{A}$; one can easily show that if $\mathcal{A}$ is a central simple associative superalgebra over a field $K$, then $\mathcal{A}^{\circ}$ is also a central simple associative superalgebra over $K$.

Definition 2.4. Let $\mathcal{A}=\mathcal{A}_{0}+\mathcal{A}_{1}, \mathbb{B}=\mathbb{B}_{0}+\mathbb{B}_{1}$ be associative superalgebras. Then the graded tensor product

$$
\mathcal{A} \widehat{\otimes}_{K} B=\left[\left(\mathcal{A}_{0} \otimes B_{0}\right) \oplus\left(\mathcal{A}_{1} \otimes B_{1}\right)\right] \oplus\left[\left(\mathcal{A}_{0} \otimes \boldsymbol{B}_{1}\right) \oplus\left(\mathcal{A}_{1} \otimes B_{0}\right)\right]
$$

where the multiplication on $\mathcal{A} \widehat{\otimes}_{K} B$ is induced by

$$
\left(a_{\alpha} \otimes b_{\beta}\right)\left(c_{\gamma} \otimes d_{\delta}\right)=(-1)^{\beta \gamma} a_{\alpha} c_{\gamma} \otimes b_{\beta} d_{\delta}, \quad a_{\alpha} \in \mathcal{A}_{\alpha}, c_{\gamma} \in \mathcal{A}_{\gamma}, b_{\beta} \in \mathbb{B}_{\beta}, d_{\delta} \in \mathbb{B}_{\delta} .
$$

If $\mathcal{A}$ and $\mathbb{B}$ are associative superalgebras, then $\mathcal{A} \widehat{\otimes}_{K} \not$ is an associative superalgebra.

The commuting super-ring of $R$ on $M$ is defined to be $\mathcal{C}=\mathcal{C}_{0}+\mathcal{C}_{1}$, where

$$
\mathcal{C}_{\gamma}:=\left\{c_{\gamma} \in \operatorname{End}_{\gamma} M \mid c_{\gamma} r_{\alpha}=(-1)^{\alpha \gamma} r_{\alpha} c_{\gamma} \forall r_{\alpha} \in R_{\alpha}, \alpha \in \mathbb{Z}_{2}\right\}
$$

Definition 2.5. Two finite dimensional central simple superalgebras $\mathcal{A}$ and $\boldsymbol{B}$ over a field $K$ are called similar $(\mathscr{A} \sim \mathcal{B})$ if there exist graded $K$-vector spaces $V=V_{0} \oplus V_{1}, W=W_{0} \oplus W_{1}$, such that $\mathcal{A} \widehat{\otimes}_{K}$ End $_{K} V \cong B \widehat{\otimes}_{K}$ End $_{K} W$ as $K$-superalgebras. 
Similarity is obviously an equivalence relation. The set of similarity classes will be denoted by BW $(K)$ (the Brauer-Wall group of $K$ ). If [A] denotes the class of $A$ in BW $(K)$ by using $\left[4\right.$, Chapter 4,Theorem 2.3(3)], the operation $[\mathcal{A}][B]=\left[\mathcal{A} \widehat{\otimes}_{K} B\right]$ is well-defined, and makes the set of similarity classes of finite dimensional central simple superalgebras over $K$ into a commutative group, BW $(K)$, where the class of the matrix algebras $M_{p+q}(K)$ is a neutral element for this product. Moreover, it was proved in $[4,5]$ that a central simple associative superalgebra $A$ is of order 2 in $\mathrm{BW}(K)$ if and only if $A \approx A^{\circ}$, the opposite superalgebra.

\section{Existence of pseudo-superinvolution on $\Phi$}

Theorem 3.1 (division superalgebra theorem [3]). If $\boldsymbol{\Phi}=\Phi_{0}+\Phi_{1}$ is a finite dimensional associative division superalgebra over a field $K$, then exactly one of the following holds where throughout $\varepsilon$ denotes a finite dimensional associative division algebra over $K$.

(i) $\Phi=\Phi_{0}=\mathcal{E}$, and $\Phi_{1}=\{0\}$.

(ii) $\Phi=\mathcal{E} \otimes_{K} K[u], u^{2}=\lambda \in K^{\times}, \Phi_{0}=\mathcal{\varepsilon} \otimes K 1, \Phi_{1}=\mathcal{\varepsilon} \otimes K u$.

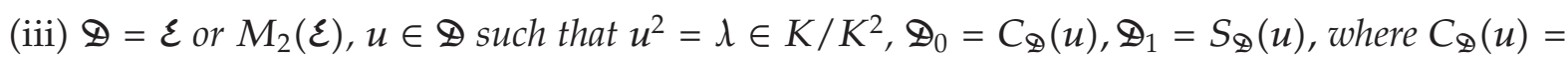
$\{d \in \Phi \mid d u=u d\}, S_{\Phi}(u)=\{d \in \Phi \mid d u=-u d\}$, moreover, in the second case, $u=\left(\begin{array}{ll}0 & 1 \\ 1 & 0\end{array}\right)$ and $K[u]$ does not embed in $\varepsilon$.

Following [4], we say that a division superalgebra $\Phi$ is even if $Z(\Phi) \cap \Phi_{1}=\{0\}$, where $Z(\Phi)$ is the center of $\Phi$, that is, $\Phi$ is even if its form is (i) or (iii), and that $\Phi$ is odd if its form is (ii). Also, if $\mathscr{A}=M_{n}(\mathscr{\Xi})$ is a finite dimensional central simple superalgebra over a field $K$, then we say that $\mathcal{A}$ is an even $K$-superalgebra if $\Phi$ is an even division superalgebra and $\mathcal{A}$ is an odd $K$-superalgebra if $\Phi$ is an odd division superalgebra.

Let $V=V_{0}+V_{1}$ be a (left) superspace over a division superalgebra $C$ and $W=W_{0}+W_{1}$ a right superspace over $\mathcal{C}$. A bilinear pairing $(,)_{v}$ is a biadditive map $(,)_{v}: V \times W \rightarrow \mathcal{C}$ satisfying

$$
\begin{gathered}
\left(v_{\alpha}, w_{\beta}\right)_{\nu} \in \mathcal{C}_{\alpha+\beta+v}, \quad\left(c_{\gamma} v_{\alpha}, w_{\beta}\right)_{\nu}=c_{\gamma}\left(v_{\alpha}, w_{\beta}\right)_{\nu^{\prime}} \\
\left(v_{\alpha}, w_{\beta} c_{\gamma}\right)_{\nu}=\left(v_{\alpha}, w_{\beta}\right)_{\nu} c_{\gamma}
\end{gathered}
$$

for all $v_{\alpha} \in V_{\alpha}, w_{\beta} \in W_{\beta}$, and $c_{\gamma} \in \mathcal{C}_{\gamma}$. The bilinear pairing $(,)_{\nu}$ is nondegenerate if

$$
\left(v_{\alpha}, W\right)_{v}=\{0\} \Longrightarrow v_{\alpha}=0, \quad\left(V, w_{\beta}\right)_{v}=\{0\} \Longrightarrow w_{\beta}=0
$$

If $(,)_{\nu}$ is nondegenerate, we say that the superspaces $V$ and $W$ are dual.

The right $\mathcal{C}$-superspace $W$ may be viewed as a (left) $\mathcal{C}^{\circ}$-superspace via

$$
c_{\gamma} w_{\beta}:=(-1)^{\beta \gamma} w_{\beta} c_{\gamma} .
$$

An element $a_{\alpha} \in \operatorname{End}_{\mathcal{C}}(V)_{\alpha}$ is said to have an adjoint $a_{\alpha}^{*} \in \operatorname{End}_{\mathcal{C}^{\circ}}(W)_{\alpha}$ if

$$
\left(v_{\beta} a_{\alpha}, w_{\delta}\right)_{v}=(-1)^{\alpha \delta}\left(v_{\beta}, w_{\delta} a_{\alpha}^{*}\right)_{v^{\prime}} \quad \forall v_{\beta} \in V_{\beta}, w_{\delta} \in W_{\delta} .
$$

Therefore, if $\boldsymbol{\Phi}$ is a division superalgebra and $\sigma$ is an antiautomorphism of $\boldsymbol{\Phi}$, then it is an isomorphism of $\Phi$ onto $\Phi^{\circ}$ and a right $\Phi^{\circ}$-superspace $W$ is a left $\Phi$ superspace under the action

$$
d_{\delta} w_{\beta}:=(-1)^{\delta \beta} w_{\beta} d_{\delta}^{\sigma}, \quad d_{\delta} \in \Phi_{\delta}, w_{\beta} \in W_{\beta} .
$$


Thus, $(,)_{v}: V \times W \rightarrow \Phi$ is a pseudo-sesquilinear pairing of (left) $\Phi$ superspaces, that is,

$$
\begin{aligned}
& \left(d_{\delta} v_{\alpha}, w_{\beta}\right)_{v}=d_{\delta}\left(v_{\alpha}, w_{\beta}\right)_{v^{\prime}} \\
& \left(v_{\alpha}, d_{\delta} w_{\beta}\right)_{v}=(-1)^{\beta \delta}\left(v_{\alpha}, w_{\beta}\right)_{v} d_{\delta^{\prime}}^{\sigma} \\
& \left(v_{\alpha} d_{\delta}, w_{\beta}\right)_{v}=(-1)^{\delta(\beta+v+1)}\left(v_{\alpha}, w_{\beta}\right)_{\nu} d_{\delta}
\end{aligned}
$$

for all $v_{\alpha} \in V_{\alpha}, w_{\beta} \in W_{\beta}, d_{\delta} \in \Phi_{\delta}$. If - is a pseudo-superinvolution of $\boldsymbol{\Phi}$, then $\boldsymbol{\Phi}$ is isomorphic to $\Phi^{\circ}$ and we may consider pseudo-sesquilinear pairings of $V \times V$. If $\epsilon \in Z(\boldsymbol{\Phi})$ with $\epsilon \bar{\epsilon}=1$, and

$$
\delta_{v}= \begin{cases}\sqrt{-1} & v=1 \\ 1 & v=0\end{cases}
$$

an $\epsilon$-Hermitian pseudo-superform is a pseudo-sesquilinear pairing satisfying

$$
\left(v_{\alpha}, w_{\beta}\right)_{v}=(-1)^{\alpha(\beta+1)} \epsilon \delta_{v} \overline{\left(w_{\beta}, v_{\alpha}\right)_{v}} \quad \forall v_{\alpha} \in V_{\alpha}, w_{\beta} \in V_{\beta}
$$

The pseudo-superform $(,)_{v}$ is said to be even or odd according to either $v=0$ or 1 . If $\epsilon=1$ (resp., -1), ( , ), is said to be Hermitian (resp., skew-Hermitian).

We say that a super-ring $R$ is prime if for any nonzero superideals $I, J$, the product $I J \neq\{0\}$. If $R=M_{n}(\Phi)$, where $\Phi$ is a division superalgebra over a field $K$, then $R$ is a prime. We also have the usual characterization for homogeneous elements:

$$
R \text { is prime } \Longleftrightarrow a_{\alpha} R b_{\beta} \neq\{0\} \quad \forall 0 \neq a_{\alpha} \in R_{\alpha}, 0 \neq b_{\beta} \in R_{\beta} \text {. }
$$

Theorem 3.2. If a central simple superalgebra $\mathscr{A}=M_{n}(\boldsymbol{\Phi}) \cong$ End $_{\boldsymbol{\Phi}}(I)$ over a field $K$ such that $\sqrt{-1} \in K$, where $I$ is a minimal right superideal of $\mathcal{A}$ and $\Phi^{\circ}$ is the commuting super-ring of $\mathcal{A}$ on $I$, has a pseudo-superinvolution $*$, then $\Phi$ has and $*$ is the adjoint with respect to a nondegenerate Hermitian or skew-Hermitian pseudo-superform on $I$.

Proof [2, Lemma 5]. $\Phi=e_{0} A e_{0}$, and $I=e_{0} \AA$ is a left $\Phi$ superspace for some symmetric primitive even idempotent $e_{0}$.

If $*$ is a pseudo-superinvolution on $\mathcal{A}$ and $e_{0}^{*}=e_{0}$, then $\left.*\right|_{\mathscr{D}}=-$ is a pseudosuperinvolution on $\Phi$, and for $v_{\alpha}=e_{0} a_{\alpha} \in I_{\alpha}, w_{\beta}=e_{0} b_{\beta} \in I_{\beta}$, define

$$
\left(v_{\alpha}, w_{\beta}\right)_{0}:=e_{0} a_{\alpha}\left(e_{0} b_{\beta}\right)^{*}=e_{0} a_{\alpha} b_{\beta}^{*} e_{0} \in \Phi_{\alpha+\beta}
$$

One checks that for all $d_{\delta} \in \Phi_{\delta}, v_{\alpha} \in I_{\alpha}, w_{\beta} \in I_{\beta}$,

$$
\begin{gathered}
\left(d_{\delta} v_{\alpha}, w_{\beta}\right)_{0}=d_{\delta}\left(v_{\alpha}, w_{\beta}\right)_{0}, \quad\left(v_{\alpha}, d_{\delta} w_{\beta}\right)_{0}=(-1)^{\beta \delta}\left(v_{\alpha}, w_{\beta}\right)_{0} \overline{d_{\delta}} \\
\left(v_{\alpha}, w_{\beta}\right)_{0}=(-1)^{\alpha(\beta+1)} \overline{\left(w_{\beta}, v_{\alpha}\right)_{0}}
\end{gathered}
$$

that $I$ is self dual with respect to $(,)_{0}$, and that $*$ is the adjoint with respect to the Hermitian pseudo-superform $(,)_{0}$. 
If the minimal right superideal $I$ contains a homogeneous $\epsilon$-symmetric element $a_{\alpha}^{*}=\epsilon a_{\alpha}$, $\epsilon= \pm 1$ such that $a_{\alpha} I \neq\{0\}$, then $a_{\alpha} I=I$, so by [2, Lemma 5], there exists an idempotent $f_{0} \in I_{0}$ such that $a_{\alpha} f_{0}=a_{\alpha}$ and $I=f_{0} \mathcal{A}$. Thus, $f_{0} a_{\alpha}=a_{\alpha}$ and

$$
a_{\alpha}=\epsilon a_{\alpha}^{*}=\epsilon\left(f_{0} a_{\alpha}\right)^{*}=\epsilon a_{\alpha}^{*} f_{0}^{*}=a_{\alpha} f_{0}^{*}=\left(a_{\alpha} f_{0}\right) f_{0}^{*} .
$$

Again the proof of [2, Lemma 5] shows that $e_{0}=f_{0} f_{0}^{*} \in I_{0}$ is a nonzero even symmetric idempotent and $I=e_{0} \mathcal{A}$ and since for $\mathcal{C}=e_{0} A e_{0}, \mathcal{C}^{\circ}$ is the commuting super-ring of $\mathcal{A}$ on $I$, $\Phi=\mathcal{C}=e_{0} A e_{0}$.

Assume from now on that if $a_{\alpha}^{*}=\epsilon a_{\alpha} \in I_{\alpha}, \epsilon= \pm 1$, then $a_{\alpha} I=\{0\}$.

We will show that if $b_{\beta} b_{\beta}^{*} \neq 0$ for some $b_{\beta} \in I_{\beta}$, then $I^{*} I=\{0\}$. Indeed, by [2, Lemma 2], $b_{\beta} b_{\beta}^{*} \neq 0$ implies that $\{0\} \neq b_{\beta} b_{\beta}^{*} \mathcal{A} \subseteq I$. Therefore, $b_{\beta} b_{\beta}^{*} \mathcal{A}=I$ and $\mathcal{A} b_{\beta} b_{\beta}^{*}=I^{*}$. Since $b_{\beta} b_{\beta}^{*} \in I$ is $\epsilon$-symmetric, $I^{*} I=\mathcal{A} b_{\beta} b_{\beta}^{*} I=\{0\}$.

We claim that $a_{\alpha}^{*} a_{\alpha}=0$ for all $a_{\alpha} \in I_{\alpha}$. Let $0 \neq a_{\alpha} \in I_{\alpha}$, by [2, Lemma 5] $I=a_{\alpha} \mathcal{A}=e_{0} \mathcal{A}$ and $\mathcal{A} e_{0}=\mathcal{A} a_{\alpha}$ is a minimal left superideal. If $b_{\beta} b_{\beta}^{*}=0$ for all $b_{\beta} \in a_{\alpha} \mathcal{A}_{\alpha+\beta}$, then we are done. Otherwise, by the preceding argument,

$$
\{0\}=I^{*} I=\mathcal{A} a_{\alpha}^{*} a_{\alpha} \mathcal{A} \quad \forall a_{\alpha} \in I_{\alpha} .
$$

Thus, $a_{\alpha}^{*} a_{\alpha}=0$, since $a_{\alpha}=a_{\alpha} r_{0}$ for some $r_{0} \in \mathcal{A}_{0}$ which implies that $a_{\alpha}^{*} a_{\alpha}=r_{0}^{*} a_{\alpha}^{*} a_{\alpha} r_{0} \in$ $\mathcal{A} a_{\alpha}^{*} a_{\alpha} \mathcal{A}=\{0\}$.

From now on, we let $I$ be a minimal right superideal of $\mathcal{A}$ such that $a_{\alpha}^{*} a_{\alpha}=0$ for all $a_{\alpha} \in I_{\alpha}$. As in [2, Lemma 5], $I=e_{0} \mathcal{A}=e_{0} \mathcal{A}_{0}+e_{0} \mathcal{A}_{1}$ and hence we have $e_{0} \mathcal{A} e_{0}^{*} \neq\{0\}$ by primeness. Therefore, $e_{0} \mathcal{A}_{v} e_{0}^{*} \neq\{0\}$ for at least one $v \in \mathbb{Z}_{2}$. We choose $v$ to be 0 , if possible. This will always be the case if $\Phi_{1}=e_{0} \mathcal{A}_{1} e_{0} \neq\{0\}$, for if $e_{0} \mathcal{A}_{1} e_{0}^{*} \neq\{0\}$, since $e_{0}^{*} \mathcal{A} e_{0}^{*}=\left(e_{0} A_{0}\right)^{*}$ is a division superalgebra, $e_{0} \mathscr{A}_{0} e_{0}^{*} \supseteq e_{0} \mathscr{A}_{1} e_{0}^{*} \mathscr{A}_{1} e_{0}^{*} \neq\{0\}$. We may therefore assume that if $v=1$, then $\Phi_{1}=\{0\}$.

Assume $e_{0} \mathcal{A}_{v} e_{0}^{*} \neq\{0\}$. If for some $r_{v} \in \mathcal{A}_{v}, r_{v}^{*}=\delta_{v} r_{v}$, then $\left(e_{0} r_{v} e_{0}^{*}\right)^{*}=\delta_{v} e_{0} r_{v} e_{0}^{*}$. If for all $r_{v} \in \mathcal{A}_{v}, r_{v}^{*}-\delta_{v} r_{v} \neq 0$, then

if $v=1$, then we have

$$
\begin{aligned}
\left(e_{0}\left(r_{v}^{*}-\delta_{v} r_{v}\right) e_{0}^{*}\right)^{*} & =e_{0}\left((-1)^{v} r_{v}-\delta_{v} r_{v}^{*}\right) e_{0}^{*} \\
& =e_{0}\left(-r_{v}-\delta_{v} r_{v}^{*}\right) e_{0}^{*} \\
& =-\delta_{v} e_{0}\left(r_{v}^{*}-\delta_{v} r_{v}\right) e_{0}^{*}
\end{aligned}
$$

if $v=0$, then we have

$$
\begin{aligned}
\left(e_{0}\left(r_{v}^{*}-\delta_{v} r_{v}\right) e_{0}^{*}\right)^{*} & =e_{0}\left(r_{v}-\delta_{v} r_{v}^{*}\right) e_{0}^{*} \\
& =-e_{0}\left(r_{v}^{*}-\delta_{v} r_{v}\right) e_{0}^{*} \\
& =-\delta_{v} e_{0}\left(r_{v}^{*}-\delta_{v} r_{v}\right) e_{0}^{*}
\end{aligned}
$$

Thus in all cases, we can choose $t_{v} \neq 0 \in \mathcal{A}_{v}$ such that

$$
\left(e_{0} t_{v} e_{0}^{*}\right)^{*}=\epsilon \delta_{v} e_{0} t_{v} e_{0}^{*}, \quad \epsilon= \pm 1
$$


Since $e_{0}^{*} \mathcal{A} e_{0} t_{v} e_{0}^{*} \neq\{0\}$, by primeness, and since $e_{0}^{*} \mathscr{A}_{0} e_{0}^{*}$ is a division algebra, one can choose $s_{v} \in \mathcal{A}_{v}$ such that

$$
e_{0}^{*} s_{\nu} e_{0} t_{v} e_{0}^{*}=e_{0}^{*}
$$

Applying *,

$$
e_{0}=(-1)^{v^{2}} e_{0} t_{v}^{*} e_{0}^{*} s_{v}^{*} e_{0}=(-1)^{v^{2}} e_{0} t_{v}^{*} e_{0}^{*} s_{v}^{*} e_{0}
$$

Therefore,

$$
\begin{aligned}
e_{0}^{*} s_{v} e_{0} & =e_{0}^{*} s_{v}\left((-1)^{v} \epsilon \delta_{v} e_{0} t_{v} e_{0}^{*} s_{v}^{*} e_{0}\right) \\
& =(-1)^{v} \epsilon \delta_{v}\left(e_{0}^{*} s_{v} e_{0} t_{v} e_{0}^{*}\right) s_{v}^{*} e_{0} \\
& =(-1)^{v} \epsilon \delta_{v} e_{0}^{*} s_{v}^{*} e_{0} .
\end{aligned}
$$

If $v=1$, then $e_{0}^{*} s_{v}^{*} e_{0}=\epsilon \delta_{v}\left(e_{0}^{*} s_{v} e_{0}\right)$. Thus

$$
\left(e_{0}^{*} s_{\nu} e_{0}\right)^{*}=\epsilon \delta_{v}\left(e_{0}^{*} s_{v} e_{0}\right) .
$$

If $v=0$, then $e_{0}^{*} s_{v}^{*} e_{0}=\epsilon \delta_{v}\left(e_{0}^{*} s_{v} e_{0}\right)$. Thus

$$
\left(e_{0}^{*} s_{v} e_{0}\right)^{*}=\epsilon \delta_{v}\left(e_{0}^{*} s_{v} e_{0}\right)
$$

So in all cases, we have

$$
\left(e_{0}^{*} s_{\nu} e_{0}\right)^{*}=\epsilon \delta_{\nu}\left(e_{0}^{*} s_{v} e_{0}\right)
$$

We therefore have

$$
\begin{aligned}
e_{0}^{*} s_{\nu} e_{0} t_{\nu} e_{0}^{*}=e_{0}^{*}, & e_{0} t_{\nu} e_{0}^{*} s_{v} e_{0}=e_{0} \\
\left(e_{0} t_{\nu} e_{0}^{*}\right)^{*}=\epsilon \delta_{\nu} e_{0} t_{v} e_{0}^{*}, & \left(e_{0}^{*} s_{v} e_{0}\right)^{*}=\epsilon \delta_{\nu} e_{0}^{*} s_{\nu} e_{0} .
\end{aligned}
$$

For $v_{\alpha}=e_{0} a_{\alpha} \in I_{\alpha}, w_{\beta}=e_{0} b_{\beta} \in I_{\beta}$,

$$
v_{\alpha} w_{\beta}^{*}=e_{0} a_{\alpha} b_{\beta}^{*} e_{0}^{*}=e_{0} a_{\alpha} b_{\beta}^{*} e_{0}^{*} s_{v} e_{0} t_{\nu} e_{0}^{*}
$$

Define

$$
\left(v_{\alpha}, w_{\beta}\right)_{v}:=e_{0} a_{\alpha} b_{\beta}^{*} e_{0}^{*} s_{\nu} e_{0} \in e_{0} \mathcal{A}_{\alpha+\beta+\nu} e_{0}=\Phi_{\alpha+\beta+v}
$$

By the last claim, $\left(v_{\alpha}, v_{\alpha}\right)_{v}:=e_{0} a_{\alpha} a_{\alpha}^{*} e_{0}^{*} s_{v} e_{0}=0$, for all $v_{\alpha} \in I_{\alpha}$. If $\left(v_{\alpha}, I\right)_{v}=\{0\}$,

$$
e_{0} a_{\alpha} \mathscr{A} e_{0}^{*} s_{v} e_{0}=\{0\}
$$

and since $e_{0}^{*} s_{\nu} e_{0} \neq 0$,

$$
e_{0} a_{\alpha}=0, \quad \text { by primeness. }
$$


Similarly, $\left(I, w_{\beta}\right)_{v}=\{0\}$ implies $w_{\beta}=0$ and $(,)_{v}$ is nondegenerate. If $d_{\delta} \in \Phi_{\delta},\left(d_{\delta} v_{\alpha}, w_{\beta}\right)_{v}=$ $d_{\delta}\left(v_{\alpha}, w_{\beta}\right)_{v}$. Moreover

$$
\begin{aligned}
\left(v_{\alpha}, d_{\delta} w_{\beta}\right)_{v} & =\left(e_{0} a_{\alpha}, d_{\delta} e_{0} b_{\beta}\right)_{v} \\
& =(-1)^{\delta \beta} e_{0} a_{\alpha} b_{\beta}^{*} e_{0}^{*} d_{\delta}^{*} e_{0}^{*} s_{\nu} e_{0}=(-1)^{\delta \beta} e_{0} a_{\alpha} b_{\beta}^{*} e_{0}^{*} s_{\nu} e_{0} t_{\nu} e_{0}^{*} d_{\delta}^{*} e_{0}^{*} s_{v} e_{0} \\
& =(-1)^{\delta \beta}\left(v_{\alpha}, w_{\beta}\right)_{\nu} e_{0} t_{\nu} e_{0}^{*} d_{\delta}^{*} e_{0}^{*} s_{\nu} e_{0}=(-1)^{\delta \beta}\left(v_{\alpha}, w_{\beta}\right)_{\nu} \overline{d_{\delta}}
\end{aligned}
$$

where

$$
\overline{d_{\delta}}:=e_{0} t_{\nu} e_{0}^{*} d_{\delta}^{*} e_{0}^{*} s_{v} e_{0}
$$

For $d_{\delta} \in \Phi_{\delta}$

$$
\begin{aligned}
\overline{\overline{d_{\delta}}} & =e_{0} t_{v} e_{0}^{*}\left(e_{0} t_{v} e_{0}^{*} d_{\delta}^{*} e_{0}^{*} s_{v} e_{0}\right)^{*} e_{0}^{*} s_{v} e_{0} \\
& =(-1)^{v^{2}+\delta} e_{0} t_{v} e_{0}^{*} s_{v}^{*} e_{0} d_{\delta} e_{0} t_{v}^{*} e_{0}^{*} s_{v} e_{0} \\
& =(-1)^{v^{2}+\delta} \epsilon \delta_{v} e_{0} d_{\delta} \epsilon \delta_{v} e_{0} \\
& =(-1)^{v^{2}+\delta}\left(\delta_{v}\right)^{2} d_{\delta}=(-1)^{\delta} d_{\delta}
\end{aligned}
$$

For $c_{\gamma} \in \Phi_{\gamma}$ and $d_{\delta} \in \Phi_{\delta}$,

$$
\begin{aligned}
\overline{c_{\gamma} d_{\delta}} & =e_{0} t_{\nu} e_{0}^{*}\left(c_{\gamma} d_{\delta}\right)^{*} e_{0}^{*} s_{\nu} e_{0}=(-1)^{\gamma \delta} e_{0} t_{\nu} e_{0}^{*} d_{\delta}^{*} c_{\gamma}^{*} e_{0}^{*} s_{\nu} e_{0} \\
& =(-1)^{\gamma \delta} e_{0} t_{\nu} e_{0}^{*} d_{\delta}^{*} e_{0}^{*} s_{\nu} e_{0} t_{\nu} e_{0}^{*} c_{\gamma}^{*} e_{0}^{*} s_{\nu} e_{0}=(-1)^{\gamma \delta} \overline{d_{\delta}} \overline{c_{\gamma}} .
\end{aligned}
$$

Thus " - " is a pseudo-superinvolution of $\Phi$ and $(,)_{\nu}$ is a nondegenerate pseudosesquilinear superform on $I$ whose adjoint is *. Finally,

$$
\begin{aligned}
\overline{\left(v_{\alpha}, w_{\beta}\right)_{v}} & =e_{0} t_{v} e_{0}^{*}\left(e_{0} a_{\alpha} b_{\beta}^{*} e_{0}^{*} s_{v} e_{0}\right)^{*} e_{0}^{*} s_{v} e_{0} \\
& =(-1)^{\alpha \beta+\beta}(-1)^{v(\alpha+\beta)} e_{0} t_{v} e_{0}^{*} s_{v}^{*} e_{0} b_{\beta} a_{\alpha}^{*} e_{0}^{*} s_{v} e_{0} \\
& =(-1)^{\alpha \beta+\beta}(-1)^{v(\alpha+\beta)} \epsilon \delta_{v} e_{0} b_{\beta} a_{\alpha}^{*} e_{0}^{*} s_{v} e_{0} \\
& =(-1)^{\alpha \beta+\beta}(-1)^{v(\alpha+\beta)} \epsilon \delta_{v}\left(w_{\beta}, v_{\alpha}\right)_{v} .
\end{aligned}
$$

If $\mathcal{v}=0$, then $\overline{\left(v_{\alpha}, w_{\beta}\right)_{0}}=(-1)^{\alpha \beta+\beta} \epsilon \delta_{0}\left(w_{\beta}, v_{\alpha}\right)_{0}$, and hence

$$
\left(w_{\beta}, v_{\alpha}\right)_{0}=(-1)^{\alpha \beta+\beta} \in \delta_{0} \overline{\left(v_{\alpha}, w_{\beta}\right)_{0}} .
$$

Thus $(,)_{0}$ is $\epsilon$-Hermitian pseudo-superform. If $v=1$, then we have assumed that $\Phi_{1}=\{0\}$ and therefore $\left(v_{\alpha}, w_{\alpha}\right)_{1}=0$, for all $v_{\alpha}, w_{\alpha} \in I_{\alpha}$. Hence the right-hand side is 0 unless $\alpha+\beta=1$. Thus for all $v_{\alpha} \in I_{\alpha}, w_{\beta} \in I_{\beta}$,

$$
\overline{\left(v_{\alpha}, w_{\beta}\right)_{v}}=(-1)^{v+\alpha \beta+\beta} \epsilon \delta_{v}\left(w_{\beta}, v_{\alpha}\right)_{v}=(-1)^{\alpha \beta+\beta} \epsilon\left(-\delta_{v}\right)\left(w_{\beta}, v_{\alpha}\right)_{v} .
$$

Thus

$$
\left(w_{\beta}, v_{\alpha}\right)_{1}=(-1)^{\alpha \beta+\beta} \epsilon \delta_{1} \overline{\left(v_{\alpha}, w_{\beta}\right)_{1}}
$$

and $(,)_{1}$ is an $\epsilon$-Hermitian pseudo-superform. 
If $\mathcal{A}=M_{n}(\boldsymbol{\Phi})$ is a finite dimensional central simple super algebra over a field $K$, where $\Phi$ is a finite dimensional division superalgebra with nontrivial grading over $K$ then, by Theorem 3.2, it is enough to study the existence of pseudo-superinvolutions on $\Phi$ to ascertain the existence of pseudo-superinvolutions on $\mathbb{A}$.

Theorem 3.3. Let $\Phi=\Phi_{0}+\Phi_{0} v$ be an even division superalgebra over a field $K$ of characteristic not 2 , then $\Phi$ has a K-pseudo-superinvolution if and only if $\Phi \approx \Phi^{\circ}$, the opposite superalgebra.

Proof. Suppose that $\Phi$ has a $K$-pseudo-superinvolution $*$, then $*$ is a $K$-antiautomorphism on $\Phi$ which implies that $\Phi \approx \Phi^{\circ}$.

Conversely, suppose that $\Phi \approx \Phi^{\circ}$, then there exists a $K$-antiautomorphism $J$ on $\Phi$. Since $J^{2}$ is a $K$-automorphism on $\Phi$, there exists $a_{\alpha} \in \Phi_{\alpha}$ such that

$$
x^{J^{2}}=a_{\alpha} x a_{\alpha}^{-1} \quad \forall x \in \boldsymbol{D}
$$

Now, $u^{J} \in Z\left(\Phi_{0}\right)=K(u)$ implies that $u^{J}=c+d u$ for some $c, d \in K$, and $u^{J} v^{J}=(v u)^{J}=$ $(-u v)^{J}=-v^{J} u^{J}$ implies that $(c+d u) v^{J}=-v^{J}(c+d u)=-(c-d u) v^{J}$, thus $c+d u=-c+d u$ implies $c=0$, and hence $u^{J}=d u, d \in K$. Moreover, $\left(u^{2}\right)^{J}=\left(u^{J}\right)^{2}$ implies that $u^{2}=d^{2} u^{2}$, so $d=1$ or $d=-1$, which means that $u^{J}=u$ or $u^{J}=-u$. So, in all cases $u^{J^{2}}=u$, thus $u^{J^{2}}=a_{\alpha} u a_{\alpha}^{-1}=u$ implies that $\alpha=0$, and hence $a_{\alpha}=a_{0} \in \Phi_{0}$.

Case(1): if $u^{J}=u$, then $\Phi_{0} \approx \Phi_{0}^{\circ}$ implies that $\Phi_{0}$ has an involution of the first kind, so by [1, Chapter 8 , Theorem 8.2], $a_{0} a_{0}^{J}=\alpha^{2}$ for some $\alpha \in K(u)$, thus $\left(a_{0} / \alpha\right)\left(a_{0} / \alpha\right)^{J}=$ $\left(a_{0} / \alpha\right)^{J}\left(a_{0} / \alpha\right)=1$. If $a_{0} / \alpha=-1$, then $a_{0}=-\alpha \in K(u)$. If not, then let $I: \Phi_{0} \rightarrow \Phi_{0}$ be a map defined by $x^{I}=\left(1+a_{0} / \alpha\right)^{-1} x^{J}\left(1+a_{0} / \alpha\right)$, an easy computation shows that $I$ is an involution of the first kind on $\Phi_{0}$, since $u^{I}=u$, and hence $x^{I}=\left(1+a_{0} / \alpha\right)^{-1} x^{J}\left(1+a_{0} / \alpha\right)$ for all $x \in \Phi$ defines a $K$-antiautomorphism of the first kind on $\Phi$, such that $x^{I^{2}}=\alpha x \alpha^{-1}$ for all $x \in \Phi$, where $\alpha \in Z\left(\Phi_{0}\right)=K(u)$.

So, we find that for the case(1) we can define a $K$-antiautomorphism (say $h$ ) such that for some $\alpha \in K(u), x^{h^{2}}=\alpha x \alpha^{-1}$ for all $x \in \Phi$, and $u^{h}=u$, and moreover, $\alpha \alpha^{h}=\alpha^{h} \alpha \in K(u)$. Suppose that $\alpha=c+d u$, where $c, d \in K$, then $v^{h^{3}}=\left(v^{h^{2}}\right)^{h}=\left(\alpha v \alpha^{-1}\right)^{h}=\left(\alpha^{-1}\right)^{h} v^{h} \alpha^{h}$, and $v^{h^{3}}=\left(v^{h}\right)^{h^{2}}=\alpha v^{h} \alpha^{-1}$, implies that $\alpha v^{h} \alpha^{-1}=\left(\alpha^{-1}\right)^{h} v^{h} \alpha^{h}$, thus $\alpha^{h} \alpha v^{h}\left(\alpha^{h} \alpha\right)^{-1}=v^{h}$, so, $\alpha^{h} \alpha \in$ $\widehat{Z}(\Phi)=K$. Therefore,

$$
(c+d u)^{h}(c+d u)=(c+d u)^{2}=c^{2}+2 c d u+d^{2} u^{2} \in K,
$$

which implies that $2 c d=0$, so $c=0$ or $d=0$, but by [3], $\Phi$ does not have a superinvolution of the first kind, implies that $d \neq 0$, hence $c=0$, therefore $\alpha=d u$. Now, $v^{h^{2}}=(d u) v(d u)^{-1}=$ $-v(d u)(d u)^{-1}=-v$, thus $h$ is a $K$-pseudo-superinvolution on $\Phi$.

Case(2): if $u^{J}=-u$, then $*: \Phi \rightarrow \Phi$ defined by $x^{*}=v x^{J} v^{-1}$ for all $x \in \Phi$ is a $K$-antiautomorphism on $\Phi$, and $u^{*}=u$, also for any $x \in \Phi, x^{* *}=b x b^{-1}$, where $b=v\left(v^{J}\right)^{-1} a_{0} \in \Phi_{0}$. Therefore, by case(1), $\oplus$ has a $K$-pseudo-superinvolution.

Theorem 3.4. Let $\Phi=\Phi_{0}+\Phi_{0} u$, where $u \in Z(\Phi)$, be a division superalgebra of odd type over $K$, then $\boldsymbol{\Phi}$ has a pseudo-superinvolution of the first kind if and only if $\sqrt{-1} \in K$, and $\boldsymbol{\Phi} \approx \boldsymbol{\Phi}^{\circ}$, the opposite superalgebra. 
Proof. Let $*$ be any pseudo-superinvolution of the first kind on $\Phi$, then $u^{*}=\alpha u$ for some $\alpha$ in $K$, so $u^{* *}=-u=(\alpha u)^{*}=\alpha^{2} u$, thus $\alpha^{2}=-1$ implies that $\sqrt{-1} \in K$.

Conversely, suppose that $\alpha=\sqrt{-1} \in K$ and $\Phi \approx \Phi^{\circ}$, then $\Phi_{0} \approx \Phi_{0}^{\circ}$, so $\Phi_{0}$ has an involution of the first kind (say $J$ ). Therefore, if $*: \Phi \rightarrow \Phi$ is defined by $(a+b u)^{*}=a^{J}+\alpha b^{J} u$, where $a, b \in \Phi_{0}$, then $*$ is a pseudo-superinvolution on $\Phi$, since

$$
\begin{aligned}
(a+b u)^{* *} & =\left(a^{J}+\alpha b^{J} u\right)^{*}=a+\alpha^{2} b u=a-b u, \\
(a u b u)^{*} & =\left(a b u^{2}\right)^{*}=(a b)^{*}\left(u^{2}\right)^{*}=-(a b)^{*}\left(u^{*}\right)^{2} \\
& =-\left(b^{*} u^{*}\right)\left(a^{*} u^{*}\right)=-(b u)^{*}(a u)^{*} .
\end{aligned}
$$

Corollary 3.5. Let $\Phi=\Phi_{0}+\Phi_{0} u$, where $u \in Z(\Phi)$, be a division superalgebra of odd type over a field $K$, such that $\alpha=\sqrt{-1} \in K$. Then the following hold.

(1) If $*$ is a pseudo-superinvolution on $\Phi$, then we can not choose $u \in \Phi_{1}$ such that $u^{*}=u$ or $u^{*}=-u$.

(2) If - is an involution of $\boldsymbol{\Phi}_{0}$, then the superalgebra $\boldsymbol{\Phi}$ has a pseudo-superinvolution $*$ extending given by

$$
(a+b u)^{*}=\bar{a}+\alpha \bar{b} u .
$$

Proof. (1) If $u^{*}=u$, then $u^{* *}=-u=u^{*}=u$, a contradiction. Also, if $u^{*}=-u$, then $u^{* *}=-u=$ $-u^{*}=u$, a contradiction.

(2) Given an involution "-" of $\Phi_{0}$, one checks that

$$
(a+b u)^{*}=\bar{a}+\alpha \bar{b} u
$$

defines a pseudo-superinvolution on the superalgebra $\Phi=\Phi_{0} \otimes K[u]$, extending " - ," such that $\left(u^{2}\right)^{*}=u^{2}$.

\section{Existence of pseudo-superinvolution on $\mathcal{A}=M_{p+q}\left(\Phi_{0}\right)$}

We say the central simple superalgebra $(\mathscr{A}, *)$ with pseudo-superinvolution is simple if the only $*$-stable superideals of $\mathcal{A}$ are $(0)$ and $\mathcal{A}$. The first lemma is a version of a standard result for super-rings with superinvolution, and the proof of this lemma is the same as the proof of [2, Lemma 11].

Lemma 4.1. If $\mathcal{A}$ is an associative super-ring with pseudo-superinvolution $*$ such that (A), *) is simple, then either $\mathcal{A}$ is simple (as a super-ring) or $\mathbb{A}=\mathbb{B} \oplus \boldsymbol{B}^{*}$, with $\boldsymbol{B}$ a simple super-ring.

In the second case, $\mathfrak{B}^{*}$ is isomorphic to the opposite super-ring $\boldsymbol{B}^{\circ}$ of $\boldsymbol{B}$. We will consider a super-ring $\mathcal{A}$ with nonzero odd part. To avoid double indices, we will write $\mathcal{A}=A+B$, where $A=\mathscr{A}_{0}$ is the even part and $B=\mathscr{A}_{1}$ the odd part. The proof of the next theorem is the same as the proof of [2, Theorem 12].

Theorem 4.2. Let $\mathcal{A}=A+B$ be an associative super-ring with $B \neq\{0\}$, and "*" a pseudosuperinvolution of $\mathcal{A}$. If $(\mathcal{A}, *)$ is simple, then either $\left(A,\left.*\right|_{A}\right)$ is simple, or

$$
A=A_{1} \oplus A_{2}, \quad B=B_{1} \oplus B_{2},
$$


where $\left(A_{i},\left.*\right|_{A_{i}}\right)$ are simple and $B_{i}$ are irreducible A-bimodules with

$$
B_{1}^{*}=B_{2}, \quad B_{2}^{*}=B_{1},
$$

such that

$$
\begin{gathered}
A_{1} B_{1}=B_{1}=B_{1} A_{2}, \quad A_{2} B_{2}=B_{2}=B_{2} A_{1}, \\
B_{1} B_{2}=A_{1}, \quad B_{2} B_{1}=A_{2}, \\
\{0\}=A_{2} B_{1}=A_{1} B_{2}=B_{1} A_{1}=B_{2} A_{2}=B_{1} B_{1}=B_{2} B_{2} .
\end{gathered}
$$

We will need more information on the pseudo-superinvolutions of $\mathcal{A}$ when the grading is not inherited from that of $\boldsymbol{\Phi}$, that is, $\boldsymbol{\Phi}=\boldsymbol{\Phi}_{0}$, and $\mathcal{A}$ is finite dimensional. If $\mathcal{A}=M_{p+q}(\boldsymbol{\Phi})$, $\mathcal{A}_{0}=M_{p}(\boldsymbol{\Phi}) \oplus M_{q}(\boldsymbol{\Phi}), p, q>0$, then we are either in that situation or in the other, described in Theorem 4.2. We consider each case in turn using the notation of Theorem 4.2.

Theorem 4.3. If $\mathscr{A}=M_{p+q}\left(\Phi_{0}\right)$, where $\mathscr{A}_{0}=M_{p}\left(\Phi_{0}\right)+M_{q}\left(\Phi_{0}\right), p, q>0$ is a finite dimensional central simple superalgebra over a field $K$ such that $\sqrt{-1} \in K$, and $*$ is a pseudo-superinvolution on $A$ and $\left(\mathscr{A}_{0},\left.*\right|_{\mathcal{A}_{0}}\right)$ is simple then $p=q, M_{p}\left(\boldsymbol{\Phi}_{0}\right)$ has an involution $\sim$ and $(\mathscr{A}, *)$ is isomorphic to $M_{2 p}\left(\Phi_{0}\right)$ with the pseudo-superinvolution $*$ given by

$$
\left(\begin{array}{ll}
a & b \\
c & d
\end{array}\right)^{*}=\left(\begin{array}{cc}
\tilde{d} & \alpha \tilde{b} \\
\tilde{\alpha} \tilde{c} & \tilde{a}
\end{array}\right),
$$

for $a, b, c, d \in M_{p}\left(\Phi_{0}\right)$, and $\alpha \in K$ such that $\alpha \tilde{\alpha}=-1$.

Conversely if $M_{p}\left(\Phi_{0}\right)$ has an involution $\sim$ then (4.4) defines a pseudo-superinvolution on the simple superalgebra $\mathcal{A}=M_{p+p}\left(\Phi_{0}\right)$ over $K$ such that $\sqrt{-1} \in K$.

Proof. Since $\mathcal{A}$ has a pseudo-superinvolution then, by Theorem 3.2, so has $\boldsymbol{\Phi}$. In this case since $\Phi=\Phi_{0}, \Phi$ has an involution "-" and $M_{p}(\boldsymbol{\Phi})$ has an involution $\tilde{a}=\bar{a}^{t}, t$ the transpose. Since $\left(\mathcal{A}_{0},\left.*\right|_{\mathcal{A}_{0}}\right)$ is simple, $M_{q}(\boldsymbol{\Phi})$ is anti-isomorphic to $M_{p}(\boldsymbol{\Phi})$ and $p=q$. Up to isomorphism, $\left(\mathcal{A}_{0},\left.*\right|_{\mathscr{A}_{0}}\right)$ is given by $\left(M_{p}(\boldsymbol{\Phi}) \oplus M_{p}(\boldsymbol{\Phi}), *\right)$ with $(a, b)^{*}=(\tilde{b}, \tilde{a})$. Letting

$$
\begin{array}{ll}
f_{11}=\sum_{i=1}^{p} e_{i i}=\left(\begin{array}{ll}
I_{p} & 0 \\
0 & 0
\end{array}\right), & f_{22}=\sum_{i=p+1}^{2 p} e_{i i}=\left(\begin{array}{ll}
0 & 0 \\
0 & I_{p}
\end{array}\right) \\
f_{12}=\sum_{i=1}^{p} e_{i p+i}=\left(\begin{array}{cc}
0 & I_{p} \\
0 & 0
\end{array}\right), & f_{21}=\sum_{i=1}^{p} e_{p+i i}=\left(\begin{array}{ll}
0 & 0 \\
I_{p} & 0
\end{array}\right) .
\end{array}
$$

We have

$$
\begin{aligned}
& \mathcal{A}_{0}=M_{p}(\Phi) f_{11} \oplus M_{p}(\Phi) f_{22}
\end{aligned}
$$

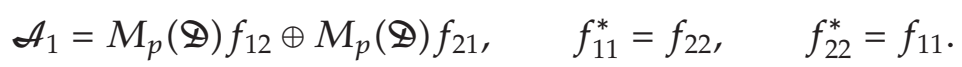

Hence

$$
\begin{aligned}
& f_{12}^{*}=\left(f_{11} f_{12} f_{22}\right)^{*}=f_{11} f_{12}^{*} f_{22}, \\
& f_{12}^{*}=c f_{12}, \text { for some } c \in M_{p}(\Phi) .
\end{aligned}
$$


For any $a \in M_{p}(\Phi)$,

$$
\left(a f_{12}\right)^{*}=\left(a f_{11} f_{12}\right)^{*}=c f_{12} \tilde{a} f_{22}=c \tilde{a} f_{12}
$$

While

$$
\left(a f_{12}\right)^{*}=\left(f_{12}\left(a f_{22}\right)\right)^{*}=\tilde{a} f_{11} c f_{12}=\tilde{a} c f_{12} .
$$

Therefore $c \in Z\left(M_{p}(\boldsymbol{\Phi})\right)$. Moreover $f_{12}^{* *}=-f_{12}=\left(c f_{12}\right)^{*}=\tilde{c} c f_{12}$ implies $\tilde{c} c=-I_{p}$. So $c=\alpha \in K$ with $\alpha \tilde{\alpha}=-1$. Similarly $f_{21}^{*}=d f_{21}, d \in Z\left(M_{p}(\boxplus)\right)$. But

$$
f_{22}=f_{11}^{*}=\left(f_{12} f_{21}\right)^{*}=-f_{21}^{*} f_{12}^{*}=-d c f_{21} f_{12}=-d c f_{22}
$$

which implies $-d c=1$, and hence $d=-c^{-1}=-\alpha^{-1}=\tilde{\alpha}$. Therefore

$$
\left(a f_{12}\right)^{*}=\tilde{a} f_{21}^{*}=\tilde{a} \tilde{\alpha} f_{21}
$$

or

$$
\left(\begin{array}{ll}
a & b \\
c & d
\end{array}\right)^{*}=\left(\begin{array}{cc}
\tilde{d} & \alpha \tilde{b} \\
\tilde{\alpha} \widetilde{c} & \tilde{a}
\end{array}\right),
$$

for $a, b, c, d \in M_{p}(\Phi)$. The converse is easy to check.

The proof of the next result is the same as [2, Proposition 14].

Theorem 4.4. If $\mathcal{A}=M_{p+q}\left(\Phi_{0}\right), p, q>0$, is a central simple superalgebra over a field $K$, and $*$ is a pseudo-superinvolution on $\mathcal{A}$, with

$$
\mathscr{A}_{0}=A_{1} \oplus A_{2}, \quad A_{1}=M_{p}\left(\Phi_{0}\right), A_{2}=M_{q}\left(\Phi_{0}\right), \quad \mathscr{A}_{1}=\mathbb{B}=\mathfrak{B}_{1}+\mathfrak{B}_{2},
$$

and $\left(\mathcal{A}_{0},\left.*\right|_{\mathcal{A}_{0}}\right)$ is not simple then $\left(A_{1},\left.*\right|_{A_{1}}\right)$ and $\left(A_{2},\left.*\right|_{A_{2}}\right)$ are simple and $\boldsymbol{B}_{i}$ are irreducible $\mathcal{A}_{0^{-}}$ bimodules with $\boldsymbol{B}_{1}^{*}=\boldsymbol{B}_{2}$ and $\boldsymbol{B}_{2}^{*}=\boldsymbol{B}_{1}$ satisfying the hypothesis of Theorem 4.2 then " $*$ " is given by

$$
\left(\begin{array}{ll}
a & b \\
c & d
\end{array}\right)^{*}=\left(\begin{array}{cc}
\tilde{a} & \tilde{c} \\
-\tilde{b} & \tilde{d}
\end{array}\right),
$$

where $a \in M_{p}\left(\Phi_{0}\right), d \in M_{q}\left(\Phi_{0}\right)$, and $\sim$ is an involution on $M_{p}\left(\Phi_{0}\right), M_{q}\left(\Phi_{0}\right)$, and where $\tilde{b} \in$ $M_{q, p}\left(\boldsymbol{\Phi}_{0}\right)$ for all $b \in M_{p, q}\left(\boldsymbol{\Phi}_{0}\right)$, and $\tilde{c} \in M_{p, q}\left(\boldsymbol{\Phi}_{0}\right)$ for all $c \in M_{q, p}\left(\boldsymbol{\Phi}_{0}\right)$.

Conversely (4.14) defines a pseudo-superinvolution on $M_{p+q}\left(\Phi_{0}\right)$.

\section{References}

[1] W. Scharlau, Quadratic and Hermitian Forms, vol. 270 of Fundamental Principles of Mathematical Sciences, Springer, Berlin, Germany, 1985.

[2] M. L. Racine, "Primitive superalgebras with superinvolution," Journal of Algebra, vol. 206, no. 2, pp. 588-614, 1998.

[3] M. L. Racine and E. I. Zelmanov, "Simple Jordan superalgebras with semisimple even part," Journal of Algebra, vol. 270, no. 2, pp. 374-444, 2003.

[4] T. Y. Lam, The Algebraic Theory of Quadratic Forms, Benjamin/Cummings, Boston, Mass, USA, 1973.

[5] C. T. C. Wall, "Graded Brauer groups," Journal für die reine und angewandte Mathematik, vol. 213, pp. 187-199, 1963/1964. 


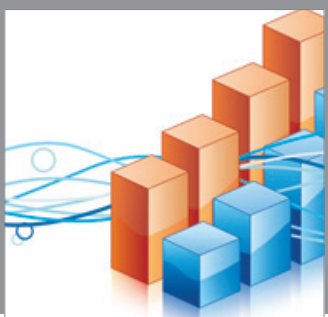

Advances in

Operations Research

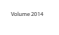

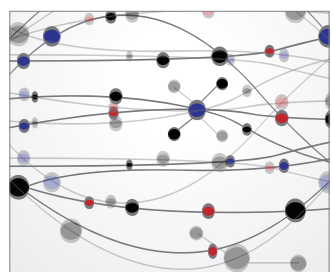

\section{The Scientific} World Journal
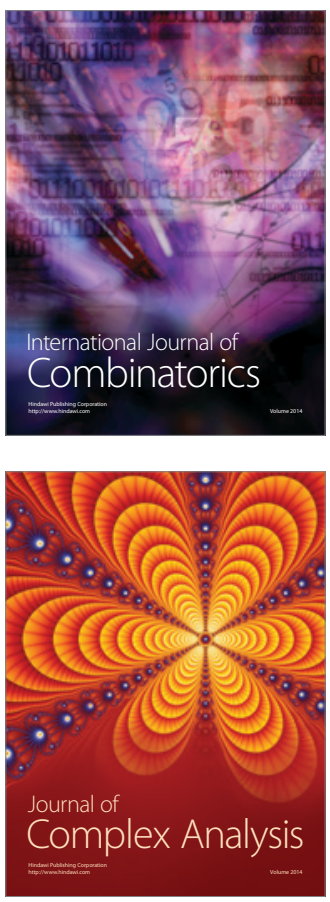

International Journal of

Mathematics and

Mathematical

Sciences
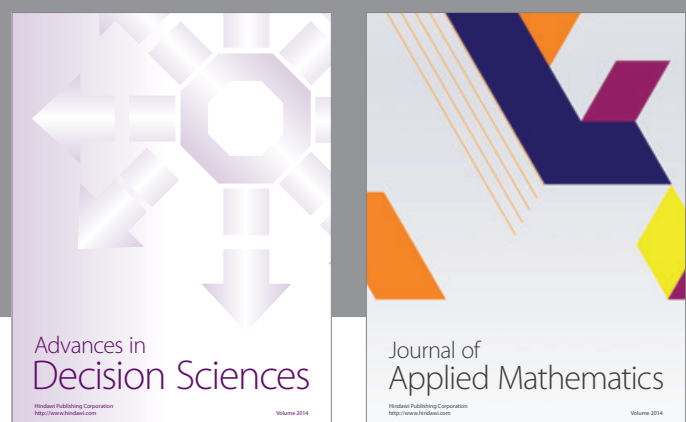

Journal of

Applied Mathematics
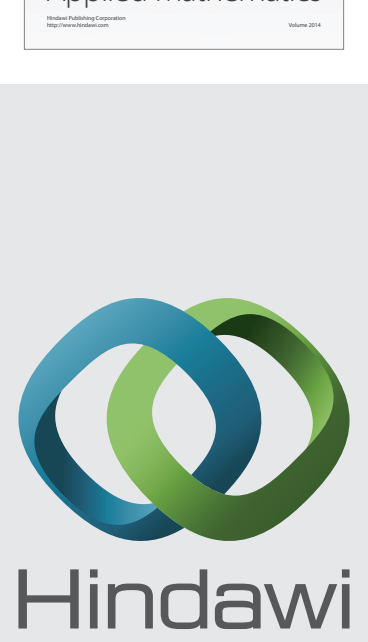

Submit your manuscripts at http://www.hindawi.com
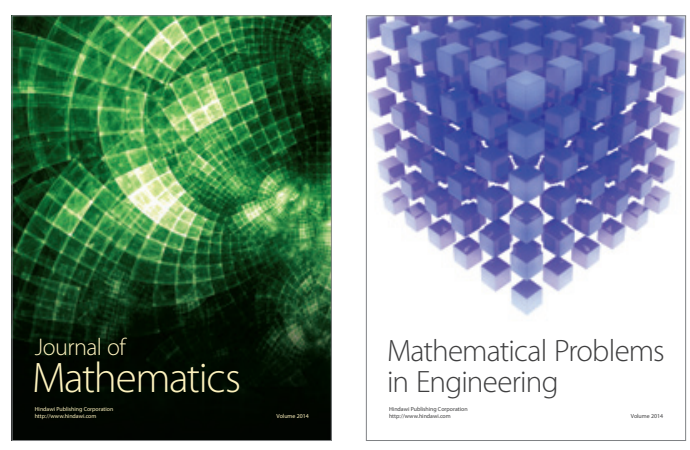

Mathematical Problems in Engineering
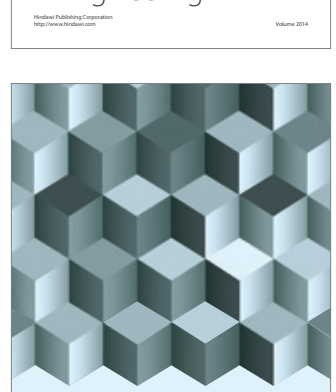

Journal of

Function Spaces
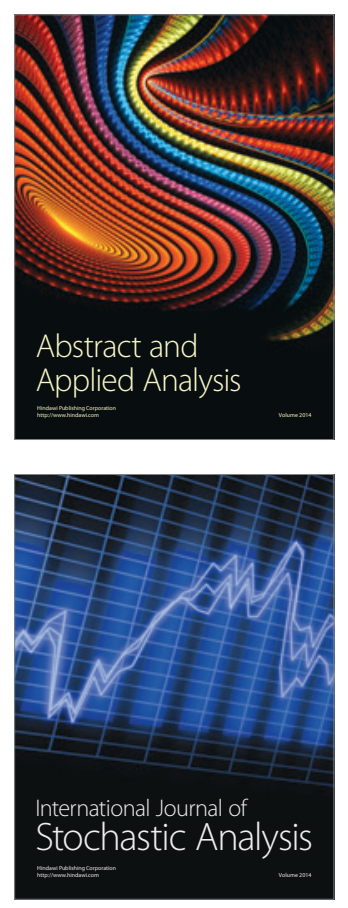

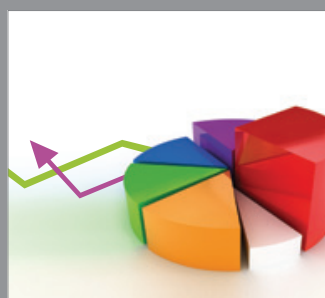

ournal of

Probability and Statistics

Promensencen
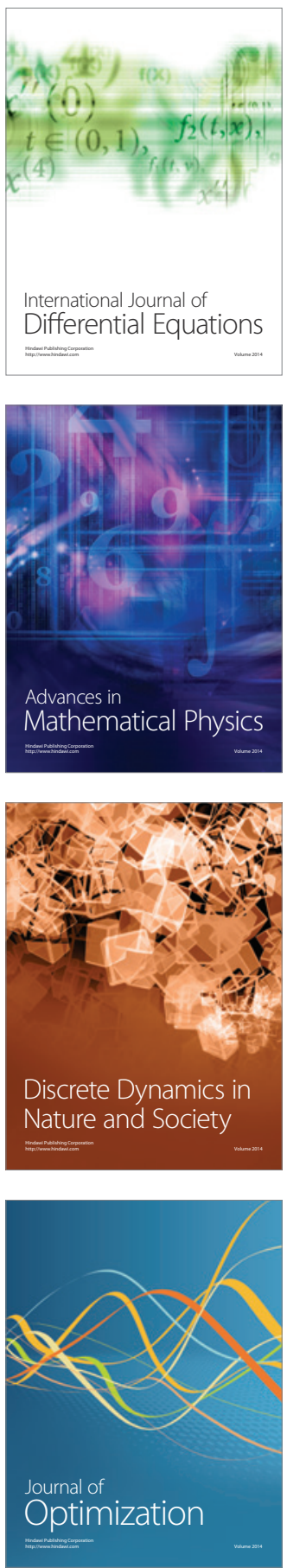